\title{
Front Accounting: An ERP for Small and Medium Agri Enterprises
}

\author{
V. V. Sumanth Kumar*, Y. Praneetha, Sanjiv Kumar and Anu Choudhary \\ ICAR-National Academy for Agricultural Research Management, \\ Rajendranagar, Hyderabad, India \\ *Corresponding author
}

\author{
A B S T R A C T
}

\begin{tabular}{l} 
Ke y w o r d s \\
Enterprise Resource \\
Planning, Front \\
Accounting, Small \\
and Medium \\
Enterprises, Multi \\
user access \\
\hline Article Info \\
$\begin{array}{l}\text { Accepted: } \\
\text { 28 February } 2020 \\
\text { Available Online: } \\
\text { 10 March } 2020\end{array}$ \\
\hline
\end{tabular}

\section{Keywords}

Enterprise Resource Planning, Front Accounting, Small user access
Agribusiness holds a large share of business niche and stands as a central element of the entire business system especially in developing economies like India. Hence, with the increasing scope for Agri-business industry, all the Agri enterprises irrespective of big, medium or small, are expanding their horizon and are bringing all the Input supply, Production, Processing, Marketing and Distribution activities under single operational management system. This requires strategic resource planning.ERP softwares, for that matter, help efficiently manage and streamline all these complex business operations and greatly enhances the productivity of the enterprise. Though SAP and ORACLE provide best ERP solutions, they are not advisable to be implemented in small and medium enterprises, for they are highly resource intensive, needs high technical expertise, highly expensive and hence not cost effective on a smaller scale. Rather, many freely available, open access software also provide ERP solutions with entirely similar functionality as those of SAP and Oracle. One such on-premise ERP platform was built and customized at ICAR-NAARM using "Front Accounting" an open access, free, web based platform, for providing ERP solution to an Agri enterprise startup "Farm Basket", an Agri input supply company. This customized platform helped the enterprise by offering a comprehensive solution for managing of materials, sales orders, finances, production, manufacturing units, supply chain and customer relationships through various modules under a single web based automated system that can be accessed by multiple users.

\section{Introduction}

In agrarian economies like India, the economic prosperity is significantly contributed by the Agri-business industry. In order to comply with the rapidly changing market conditions, Agri business enterprises are adopting digital information systems which help leverage the technology to build smart and effective business management strategies. Enterprise Resource Planning (ERP) is one such system that offers a comprehensive solution and it streamlines all the complex business functions viz. Sales and Purchase monitoring, Financial Management, Supply chain Management, Manufacturing and Resource Planning, Human Resource Management and Customer Relation Management of various operational units into a single integrated system. While many large 
scale enterprises are investing heavily in digital tools and technologies like SAP, Oracle etc., in the process of their digital transformation strategies and for ERP solutions, small and medium enterprises can rely upon many freely available open access softwares which provide ERP solutions with entirely same features as those of large scale enterprises.

In this instance, this article describes how an ERP platform is designed for a medium scale Agri-business startup "Farm Basket", based upon "Front Accounting" a free, open access professional web-based Accounting system for the entire ERP chain written in PHP, using MySQL. FA supports multicurrency and multilingual interface making it possible for the enterprise in expanding the business across the globe. By implementing the customized ERP, the enterprise is able to automate all the business operations with the help of modular architecture provided, where in specific configurations are possible for every particular group of stakeholders under each module. "Farm Basket" is a local based innovative Agri business startup dealing with the supply of all inputs related to farm ranging from different varieties of seeds of several Horticultural and Field crops, Forage seeds, Plant Protection Chemicals, Plant Nutrients and Farm Machinery.

Therefore, the enterprise has to simultaneously look after Sales and Purchase Monitoring, Financial administration, Inventory management, Distribution and also the most important Customer relationship management as well. Hence, a customized ERP system was designed (https://pgdma.in/anu/) and the configurations were done under each specific module.

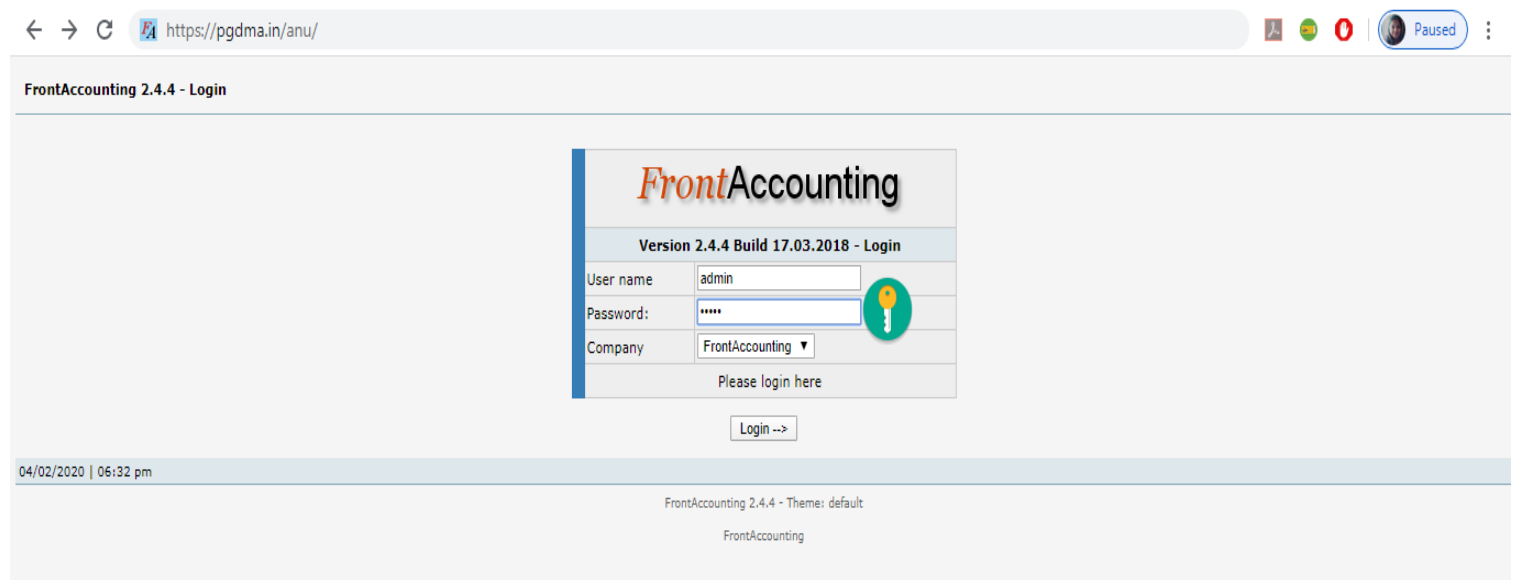

All the modules provided in the ERP platform share a single cloud powered database system. Cloud computing would enable a company's accounts and other financial information to be stored and run in the cloud itself thus, preventing it from theft or any other kind of threat. The dashboard can be accessed by all the authorized users under various departments in the enterprise so that the employee coordination is also improved due to information accuracy and quick availability. This platform, thus enables the enterprise administrators to monitor the capacity and utilization efficiency of both Material and Human resources simultaneously. Also, cloud powered platform provides them with intelligent reports generated automatically.

\section{Various modules in the customized ERP platform}

The various modules in the customized ERP platform is depicted in the figure below: 


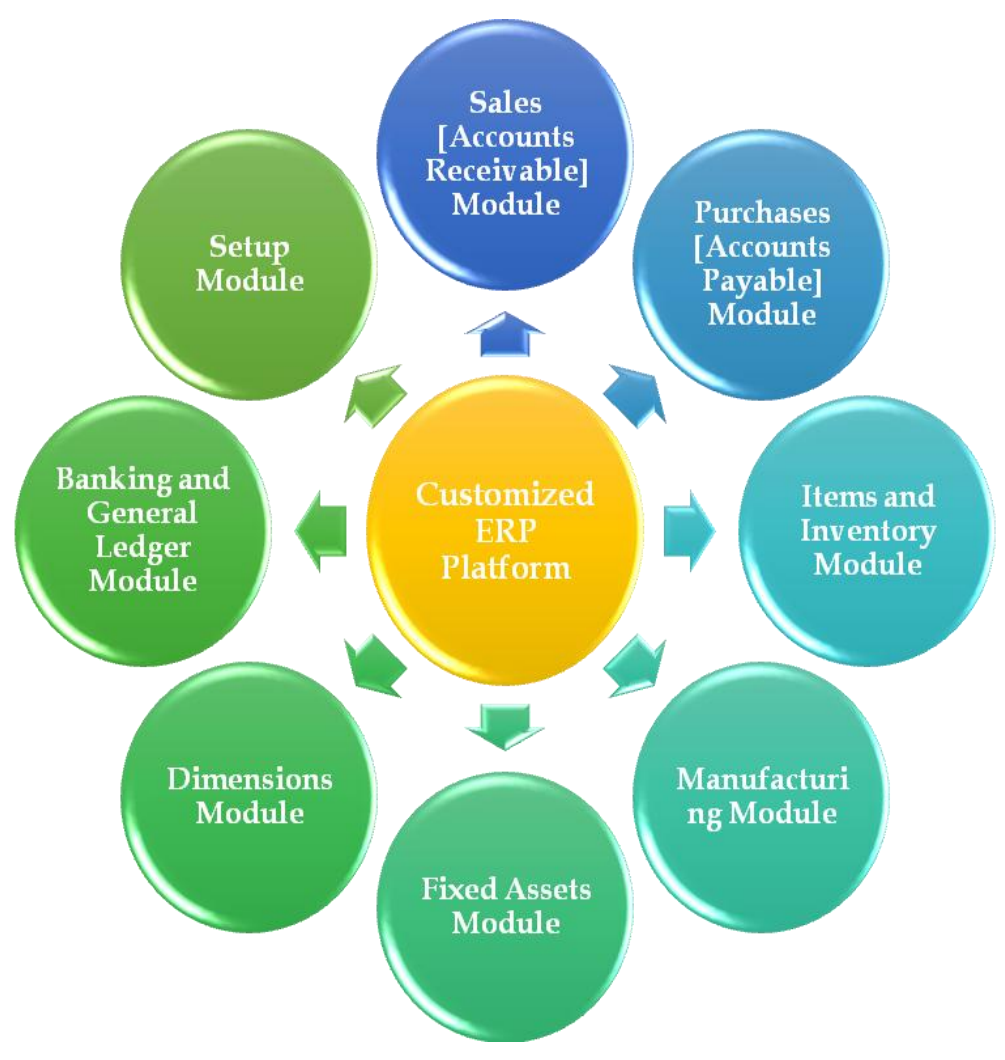

\section{Sales [Accounts Receivable] Module}

Customers are configured under Sales module. It includes the data regarding the details of the customers along with their transactions history. This module assists the entrepreneurs in assessment of sales performance and thus help them plan clever marketing and discount strategies. The entire documentation right from receiving orders from the customers until the final physical movement of the goods to customers, is done and stored under this module i.e., providing a Quote for the customers as per price of the goods demanded and allowing further negotiations and requoting if necessary; creating sales order for future transactions, and then creating invoices both for the prepaid orders as well as to make request for the payments to be received. In this instance, different customer groups for different commodities like seeds, machinery, plant protection chemicals and plant nutrients can be categorically listed under maintenance subsystem within the sales module.

\section{Purchases [Accounts Payable] Module}

Suppliers are configured under this module. This module streamlines the entire procurement function of the enterprise and helps the owners in assessment of procurement efficiency through tracking of suppliers' statistics, comparative price for the goods required and discounts available. The entire operations of the supply chain management of the enterprise are automated and integrated under this single module. It covers tracking and documenting all the operations involved in procurement right from giving order to the supplier to until final physical procurement of the goods viz. ordering for the required quantities of various commodities from different vendors, receiving invoice from the suppliers, then the Goods Receipt Note (GRN) which serves as an evidence for the goods received. Also, the automated reports generated helps the administrators track all the supplier transactions along with the outstanding purchases. 


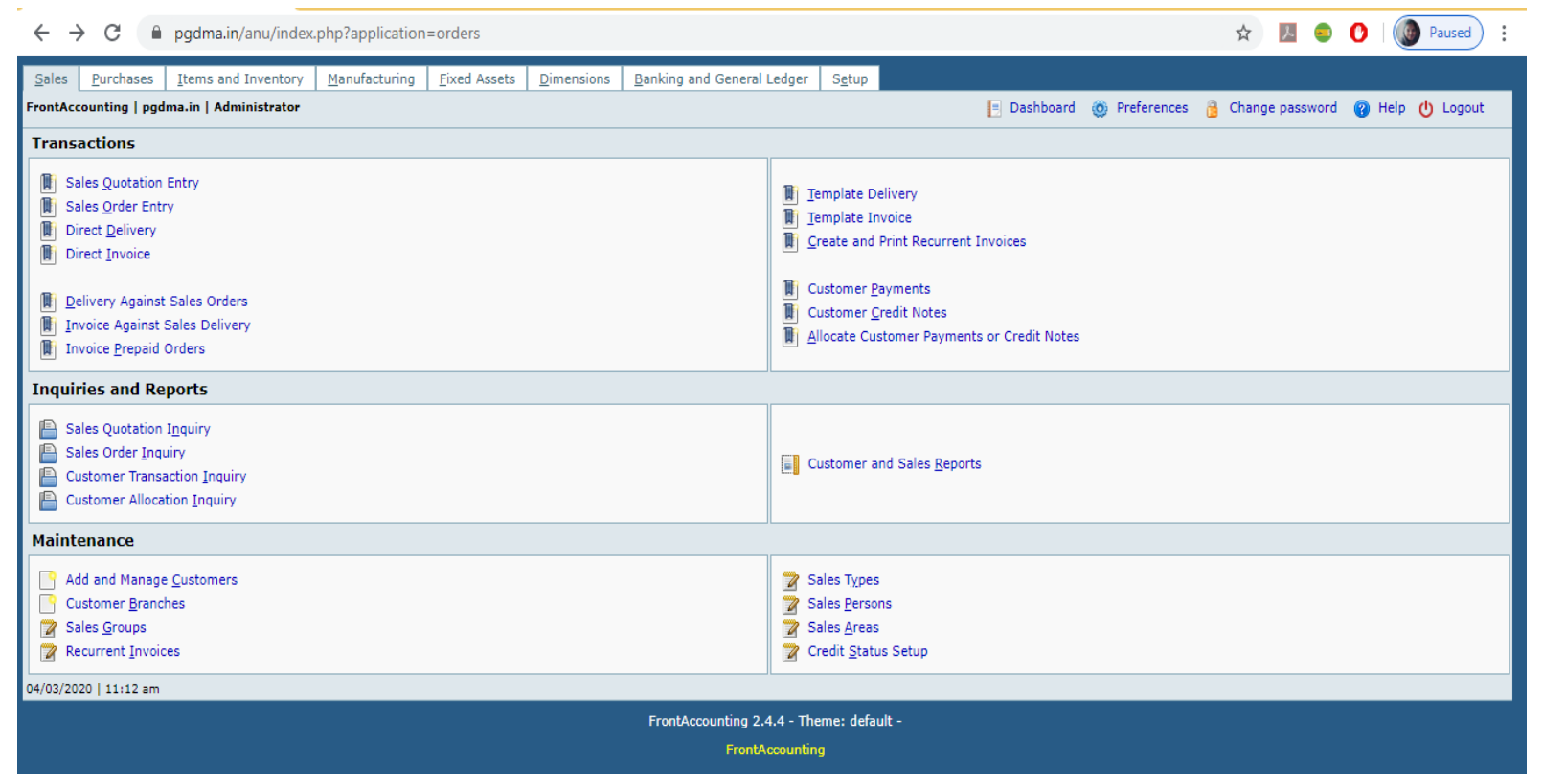

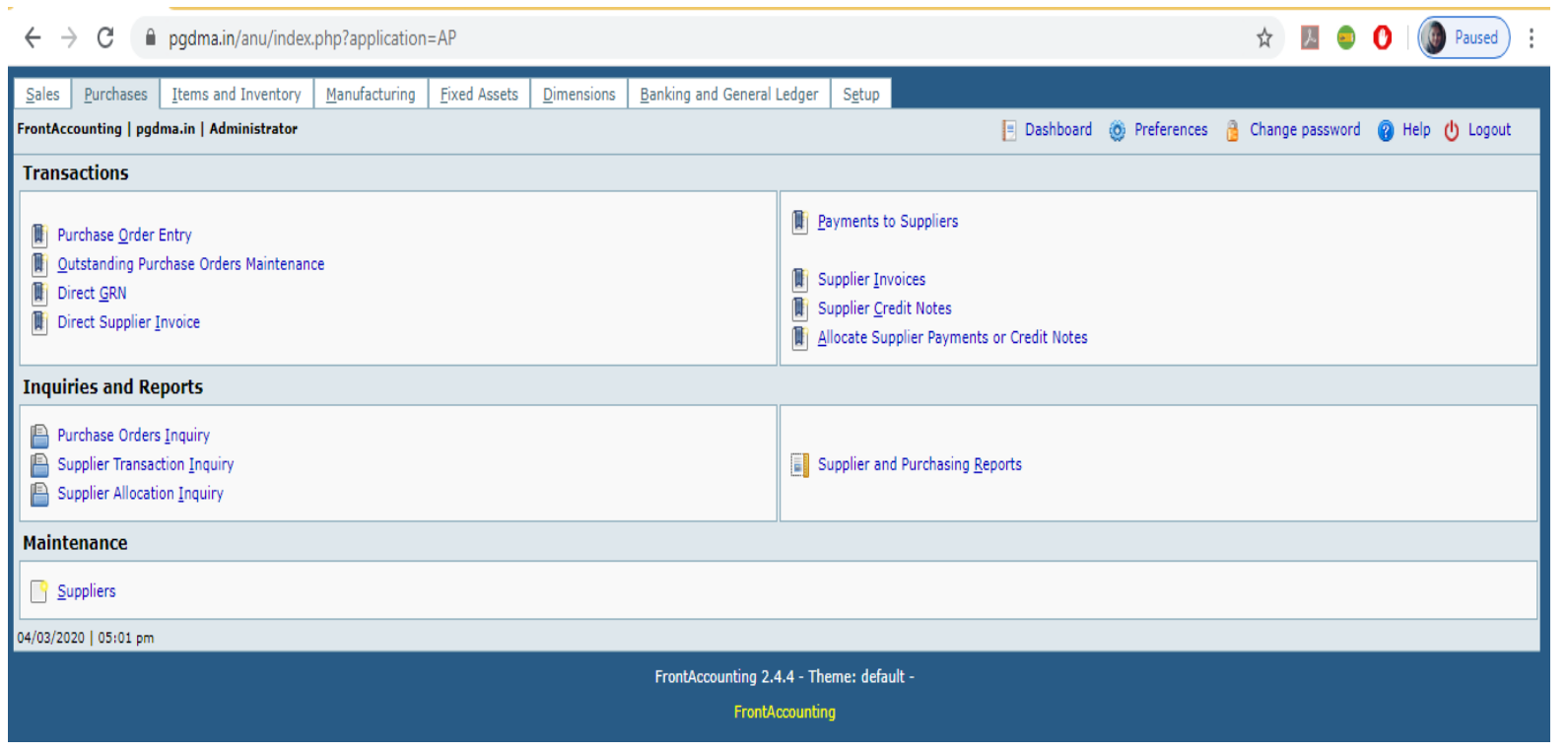

\section{Items and Inventory Module}

This module streamlines all the operations related to Warehouse management function of the enterprise and helps the administrators in maintaining record for Stock items in the enterprise. It facilitates to add different items in stock and set specific standard units of measure for different commodities and also categorize them into separate groups under Maintenance panel. It also assists in recording inventory location transfers and other adjustments if floor stock storage location is changed either to a place within the enterprise or to other locations. Besides, it also helps to set re-order levels for Inventory Items based on the availability. The other automated and additional features under this module include calculation of average material cost (per unit), Application of standard and overhead costs into material prices and Foreign codes registration for barcode scanner entry. 


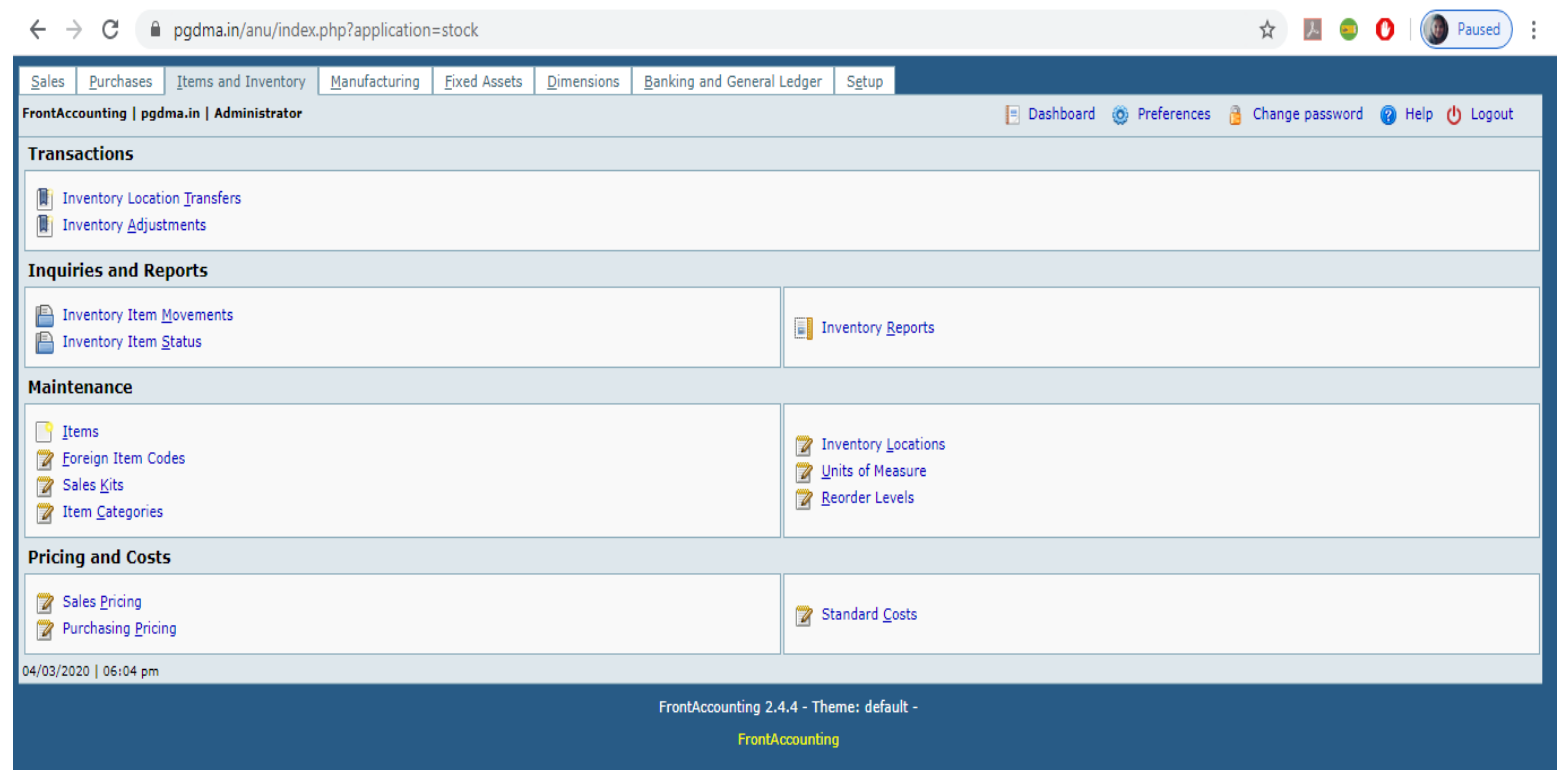

\section{Manufacturing Module}

The operations like preparation of bills for materials, work center inquiries and work order entries are streamlined under this module. With the assessment of work centers capability enterprise planners would be able to plan alternatives to meet several customer needs on time. Under this module, there is feasibility for record keeping digitally for all the activities right from simple assembling to advanced production operations. Department wise reports need to be customized under this module with either location in the inventory for the stock or the work centers in the manufacturing module for the finished goods can be used. In this instance, the down payments whether received in case of sale of inputs or to be paid in case of procurement of inputs are usually done in phases and invoices are generated in those specific modules accordingly and all these are decided as per the status in this module.

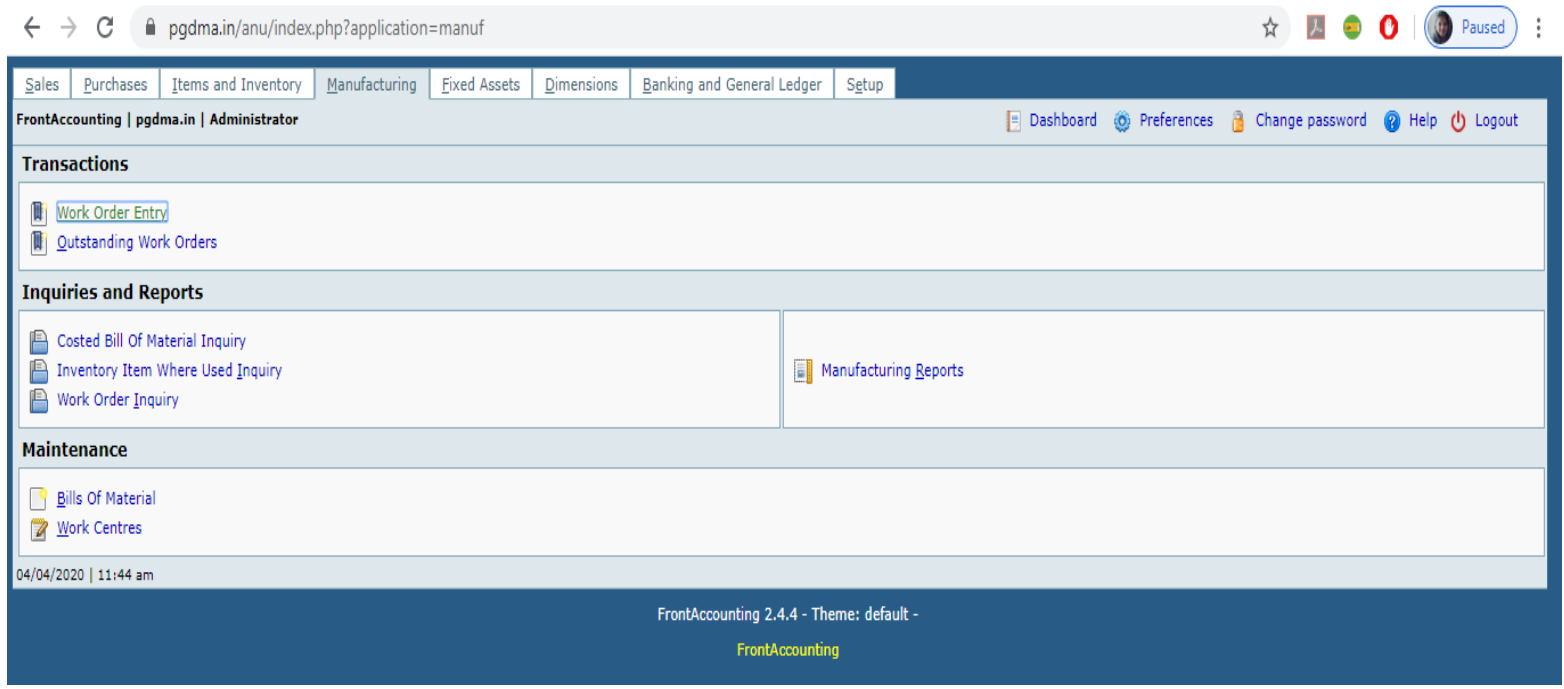




\section{Fixed Assets Module}

This module allows for registering the fixed assets owned by the enterprise. It also allows for defining the assets into several classes, categories and locations and also facilitates to record the transactions made on those assets in terms of either disposal or purchasing and also location transfers. Also this facilitates for calculation of Depreciation cost incurred to the enterprise on several fixed assets as per the standard base rates.

\section{Dimensions Module}

Usually, Dimensions are used to track departments, cost/profit/investment centers or projects within an enterprise. For example, in this instance, the enterprise has to track various departments like Sales, Procurements, Customer Service, Marketing etc., Usually dimensions are divided into separate levels viz. 0,1 and 2 based on no. of types to be tracked. But for this instance, it is only one default dimension as we need to track only several departments as the enterprise doesn't include Research and Development division. It is possible to view outstanding dimensions and also assign standard documents against a particular dimension.

$\leftarrow \rightarrow C \quad$ pgdmain/anu/index.php?application=proj

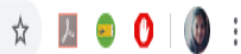

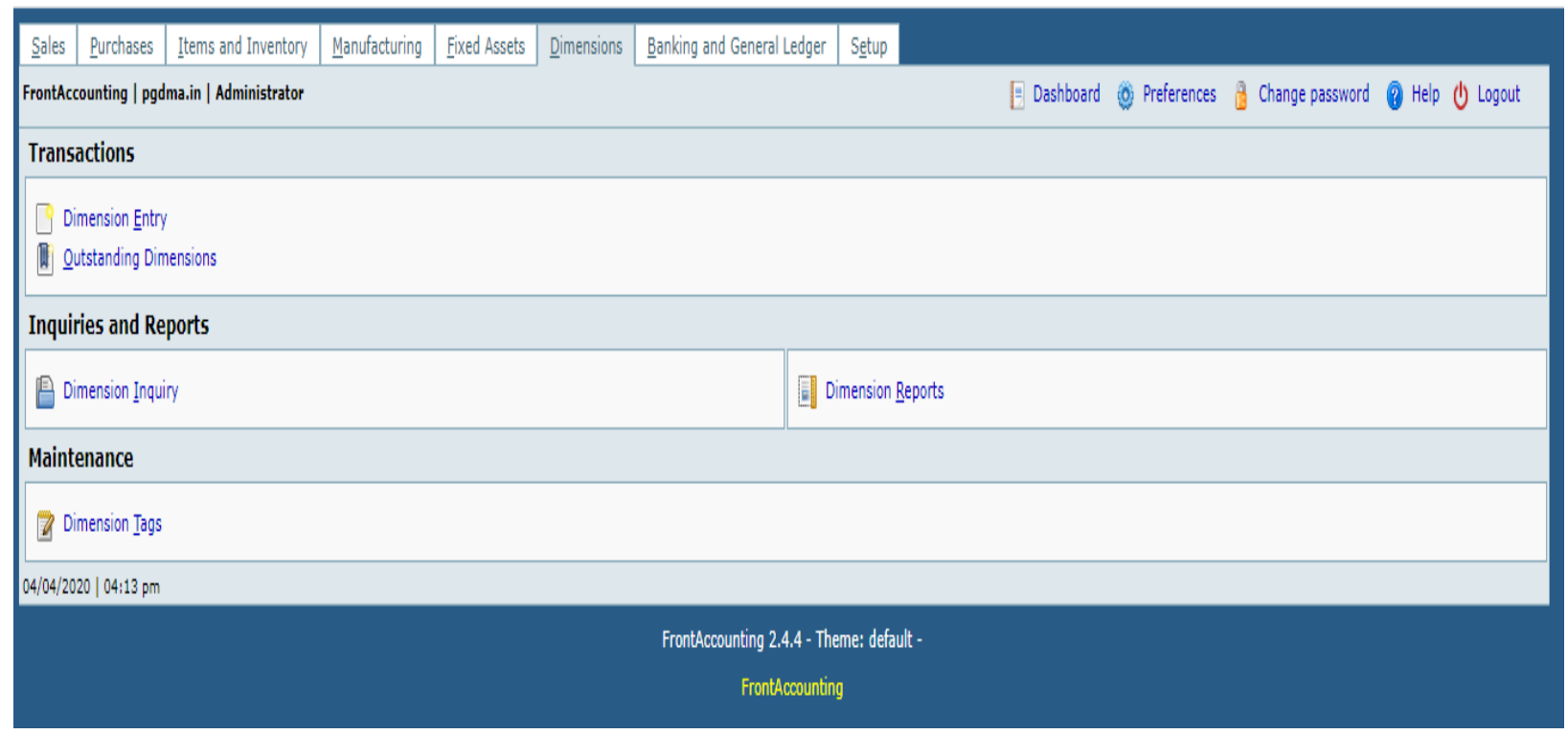

\section{Banking and General Ledger Module}

This module streamlines all the operations related to Financial Accounting functions of the enterprise. Under this module, Open bank and Petty cash accounts can be maintained and also possible to record any transactions (payments, deposits, withdrawals) done through those accounts. Automated reports are generated after the fiscal year and helps in assessment of the enterprise on financial terms. The most beneficial feature is that it also allows to create back dated transaction hence enables to maintain entire record of transactions digitally since the starting of the enterprise. This module serves as a great possibility for accounts reconciliation. Also, it is possible for automated foreign currency translations as per standard exchange rates. The database generates reports automatically based on all the operations performed and thus helps for quick reference and assessment. 


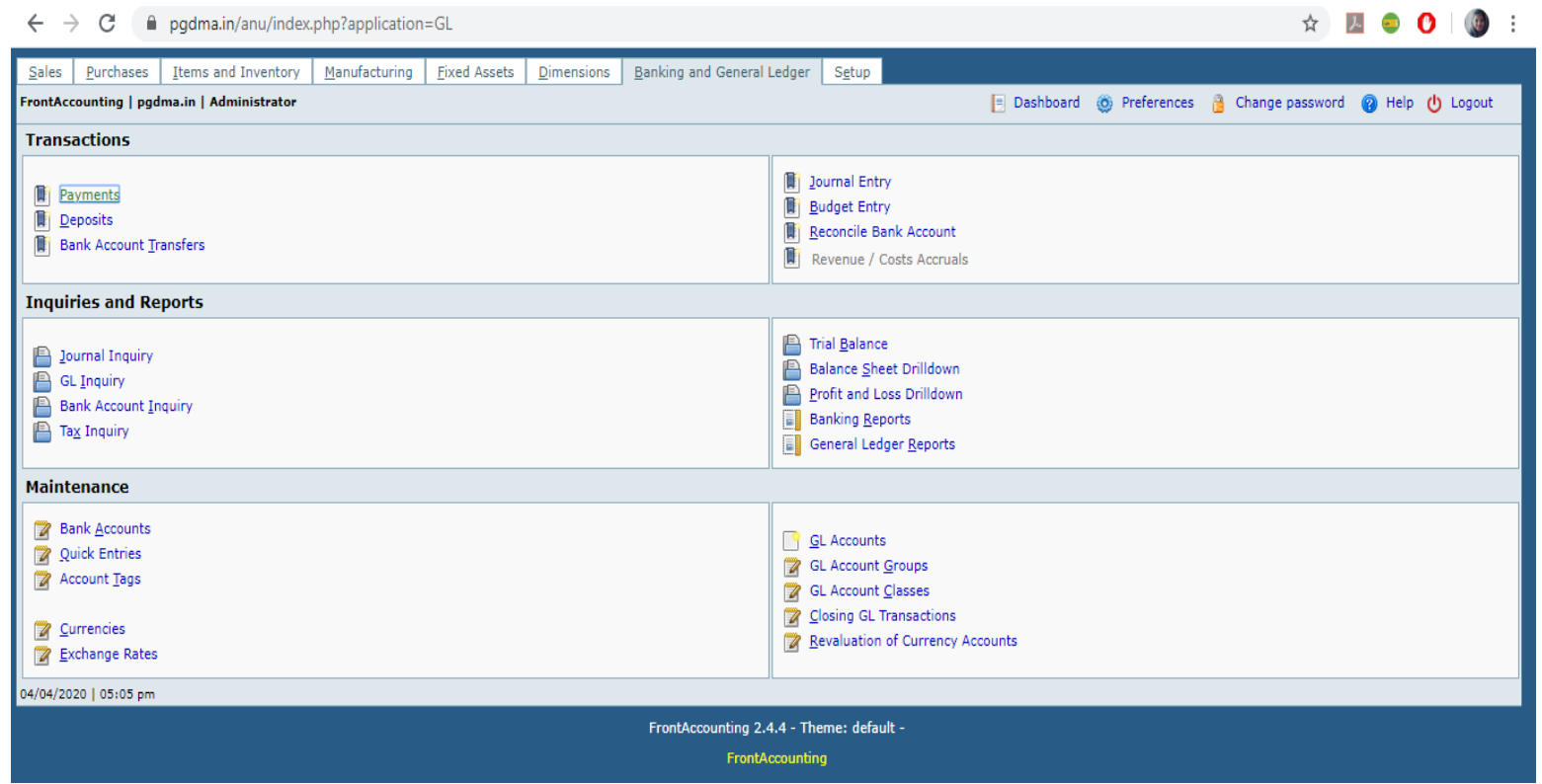

\section{Setup Module}

Under this module, it is possible to set-up different companies simultaneously. The module facilitates multi user access with different privileges and also facilitates for backing up and restoration of data. It enables for customization of menu tabs and creating extensions to suit to the specific enterprise function. It is possible for quick search due to date picker option with week numbers for all date fields. Most importantly it facilitates for periodic monitoring of the enterprise functioning through carrying out simple audit trails from time to time.

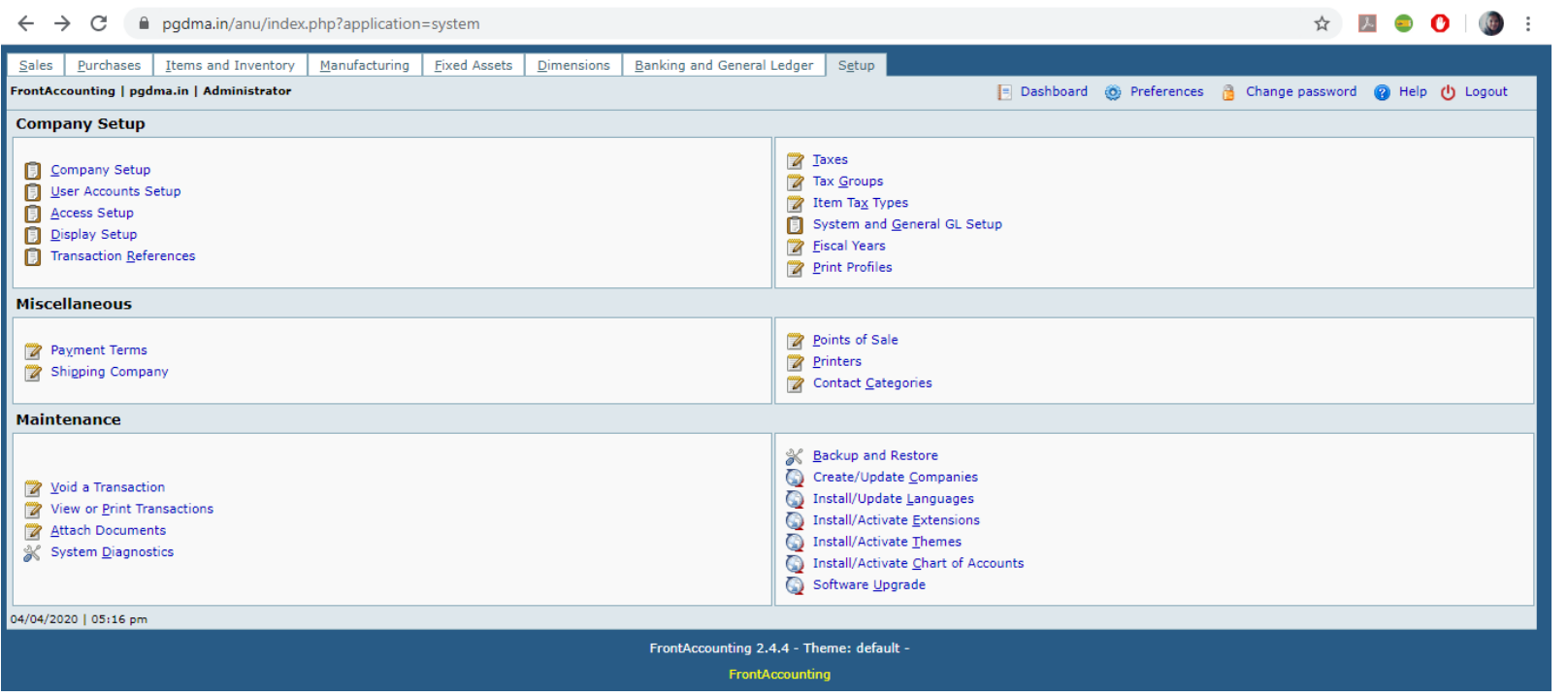

Cloud Powered ERP- Enterprise workflow

The entire enterprise workflow through this Cloud powered ERP system can be summarized as: Initially, upon receiving sales order from the customers, it is checked with the planning side of Manufacturing department for details of availability of stock of the raw 
materials and other goods. In case the stock is limited or not available, purchasing requisition $(\mathrm{PR})$ is initiated against quotations are taken from the respective vendors. After choosing the quotation from selected vendor of choice, purchasing order (PO) is created to buy material for sales order created earlier and a bill against purchasing order is sent to the Finance department. After paying bill, the material is procured from the vendor. Thus after procurement, inventory department issues material for sales order. The order is then finally dispatched to the customer after few other activities like further processing and enterprise labeling and then sales invoice is generated. The customer completes the payment against sales invoice to finance department. In this way, the sales and purchases are recorded in the finance department. On the basis of purchase order and sales invoice, the accounting and book keeping processes are done by this system automatically.

In conclusion, it is witnessed that, integration of this customized, cloud powered ERP platform within the enterprise has largely improved the business performance through time and money saving besides reducing the resource wastage to a greater extent. The enterprise could also establish greater coordination among the employees and also strengthened relationships with its customers and suppliers. Hence, it is highly advisable that other SMEs also integrate such freely accessible and scalable ERP solutions and realize greater performance and profitability.

\section{References}

1. About Front Accounting at https://frontaccounting.com/

2. Ignatio Madanhire., Charles Mbohwa., (2016). Enterprise resource planning (ERP) in improving operational efficiency: Case study. Procedia CIRP.40: 225-229.

3. Verdouw C.N., Robbemond, R.M., and Wolfert J. (2015). ERP in Agriculture: Lessons learned from Dutch Horticulture. Computers and Electronics in Agriculture.114:125-133.

4. Ahmad T., Ahmad S., and Mohammed J., (2015). A Knowledge based Indian Agriculture: with Cloud ERP Arrangement. International Conference on Green computing and Internet of Things. https://scihub.tw/10.1109/ICGCIoT.2015.7380484

\section{How to cite this article:}

Sumanth Kumar, V. V., Y. Praneetha, Sanjiv Kumar and Anu Choudhary. 2020. Front Accounting: An ERP for Small and Medium Agri Enterprises. Int.J.Curr.Microbiol.App.Sci. 9(03): 3287-3294. doi: https://doi.org/10.20546/ijcmas.2020.903.377 